\title{
Two Cases of Pediatric AQP4-Antibody Positive Neuromyelitis Optica Spectrum Disorder Successfully Treated with Tocilizumab
}

\author{
Markus Breu ${ }^{1}$ Sarah Glatter ${ }^{1}$ Romana Höftberger ${ }^{2}$ Michael Freilinger ${ }^{1}$ Karl Kircher $^{3}$ \\ Gregor Kasprian ${ }^{4}$ Rainer Seidl ${ }^{1}$ Barbara Kornek ${ }^{5}$
}

${ }^{1}$ Department of Pediatric and Adolescent Medicine, Medical University of Vienna, Vienna, Austria

2 Institute of Neurology, Medical University of Vienna, Vienna, Austria

${ }^{3}$ Department of Ophthalmology and Optometrics, Medical University of Vienna, Vienna, Austria

${ }^{4}$ Department of Biomedical Imaging and Image-guided Therapy, Medical University of Vienna, Vienna, Austria

${ }^{5}$ Department of Neurology, Medical University of Vienna, Vienna, Austria

Neuropediatrics 2019;50:193-196.

\begin{abstract}
Address for correspondence Rainer Seidl, MD, Department of Pediatric and Adolescent Medicine, Medical University of Vienna, Währinger Gürtel 18-20, 1090 Vienna, Austria

(e-mail: rainer.seidl@meduniwien.ac.at).
\end{abstract}

\author{
Abstract \\ Keywords \\ - neuromyelitis optica \\ - tocilizumab \\ - pediatric \\ - AQP4 \\ - interleukin-6 \\ - plasmablasts
}

B cell depletion with the anti-CD20-antibody rituximab is widely considered treatment of choice for long-term immunotherapy in aquaporin-4 (AQP4)-antibody positive neuromyelitis optica spectrum disorder (NMOSD). However, up to $30 \%$ of patients suffer from relapses despite complete B cell depletion. In these cases, the IL6 (interleukin-6)-receptor blocking antibody tocilizumab has been suggested as an alternative. We report two female adolescents with AQP4-antibody positive NMOSD who relapsed under rituximab treatment and clinically stabilized after switching to monthly administrations of tocilizumab. Our data suggest that early escalation of therapy with tocilizumab may lead to stabilization of disease activity in pediatric NMOSD patients who relapse under B cell depletion.

\section{Introduction}

Neuromyelitis optica spectrum disorder (NMOSD) is a rare autoimmune disease of the central nervous system that predominately affects the optic nerves and spinal cord. Serum antibodies against the astrocytic aquaporin-4 antigen (AQP4abs) are considered important factors of pathogenesis. Acute treatment of relapses includes high-dose intravenous methylprednisolone (IVMP), IV immunoglobulins (IVIG), and plasma exchange. For long-term immunotherapy and relapse prophylaxis oral corticosteroids and immunosuppressive drugs, such as azathioprine or mycophenolate mofetil have been proposed, yet, B cell depletion with the chimeric anti-CD20-antibody rituximab is widely considered treatment of choice. However, up to $30 \%$ of patients suffer from clinical relapses in spite of complete B cell depletion as measured by flow cytometric analysis. ${ }^{1-3}$ For those patients the treatment with the interleukin-(IL)-6-receptor blocking antibody tocilizumab has been proposed as a viable alternative. ${ }^{4}$ We report two female adolescents who were treated with tocilizumab after relapsing under B cell depletion.

\section{Case 1}

A previously healthy 17-year-old girl presented with hypesthesia of both hands, proprioception deficits in both legs, and urinary retention. Spinal magnetic resonance imaging (MRI) showed longitudinally extensive transverse myelitis (LETM) from C1 to the conus; cranial MRI revealed no abnormalities (-Fig. 1). Cerebrospinal fluid (CSF) studies showed a mild pleocytosis with slightly increased protein, received

September 11, 2018

accepted after revision

February 4, 2019

published online

March 26, 2019 (c) 2019 Georg Thieme Verlag KG Stuttgart · New York
DOI https://doi.org/ 10.1055/s-0039-1684004. ISSN 0174-304X. 

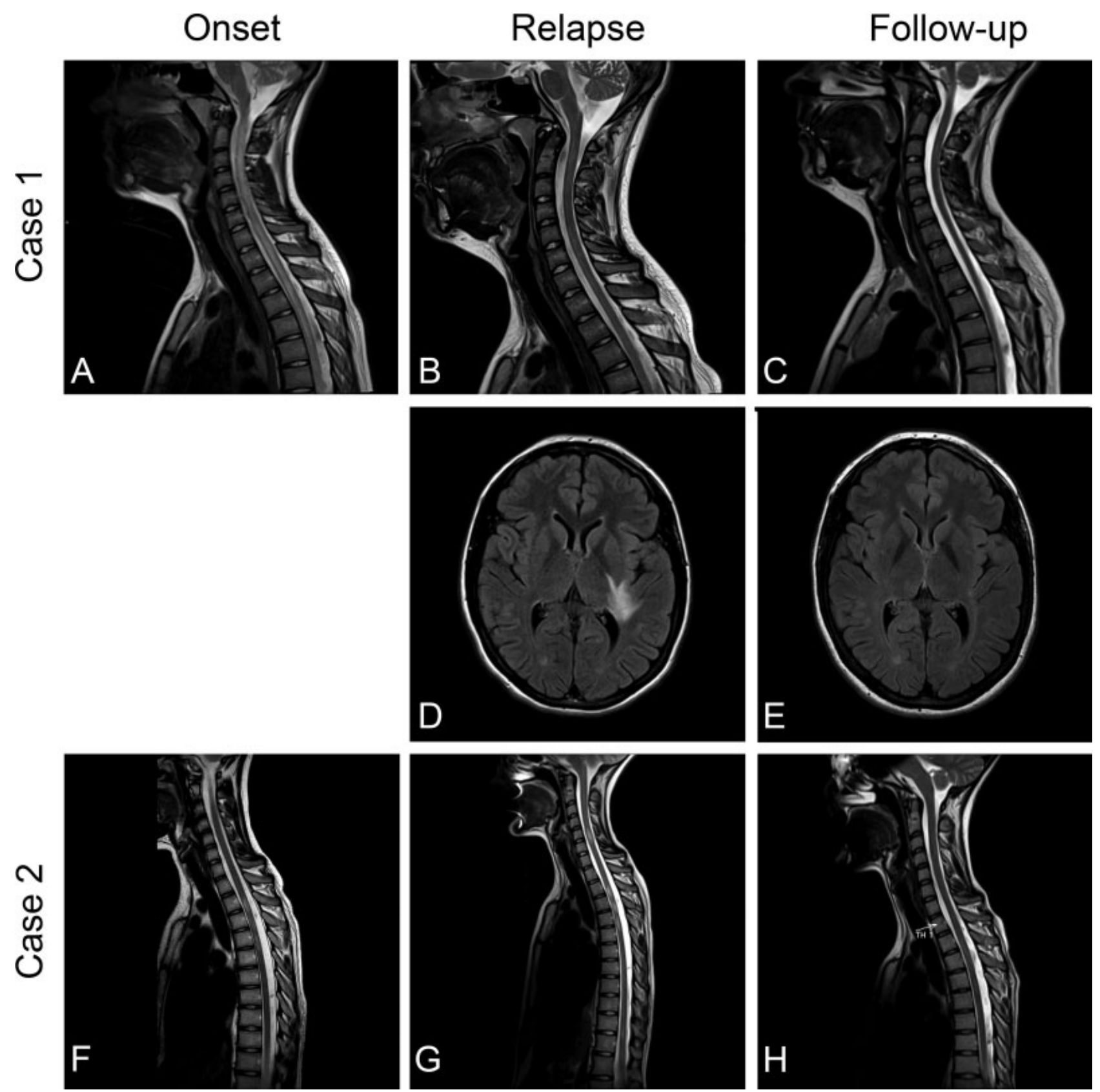

Fig. 1 MRI at onset, relapse, and follow-up in two pediatric NMOSD patients treated with tocilizumab after relapse. Sagittal spinal T2 images of case 1 (A, B, C) showing resolution of LETM until follow-up. Axial cranial T2 images of case 1 (D, E) showing resolution of a T2-hyperintense lesion in the left centrum semiovale. Sagittal spinal T2 images of case $2(\mathrm{~F}, \mathrm{G}, \mathrm{H})$ showing increase of LETM at relapse and subsequent resolution until follow-up. LETM, longitudinally extensive transverse myelitis; MRI, magnetic resonance imaging; NMOSD, neuromyelitis optica spectrum disorder.

decreased glucose, and negative oligoclonal bands. Symptoms remitted under treatment with IVMP (30 mg/day for 5 days), acyclovir, and ceftriaxone. After 3 months the patient presented with a relapse of urinary retention, this time hypesthesia extended to multiple areas of the pelvis, soles, face, and inner thighs. In addition, significant fatigue, bilateral vision field deficits, and pain on movement of both eyes (right $[R]>$ left [L]) was reported (expanded disability status scale [EDSS] 4.0). MRI showed multiple new lesions in centrum semiovale and multiple new spinal lesions ( - Fig. $\mathbf{1}$ ) as well as optic nerve enlargement, T2 hyperintensity, and contrast enhancement (-Fig. 2). Antibody testing revealed AQP4-abs in serum
$(1: 20,480)$. The patient was successfully treated with IVMP, IVIG, plasma exchange (PLEX, 10x) and oral prednisolone tapering. Subsequently, therapy with rituximab $\left(375 \mathrm{mg} / \mathrm{m}^{2}\right.$ body surface are [BSA], 2 weeks interval) was initiated. Three months later the patient presented with relapse of vision field deficits on the left side without changes in visual acuity while under complete B cell depletion as measured by flow cytometric analysis. The patient was treated with IVMP, IVIG, and PLEX (7x). Long-term therapy with tocilizumab $(8 \mathrm{mg} / \mathrm{kg}$, monthly) was started, which led to clinical stabilization. Optical coherence tomography (OCT) revealed atrophy of both optic nerves 4 months later $(\mathrm{L}>\mathrm{R})$. 


\section{Onset}

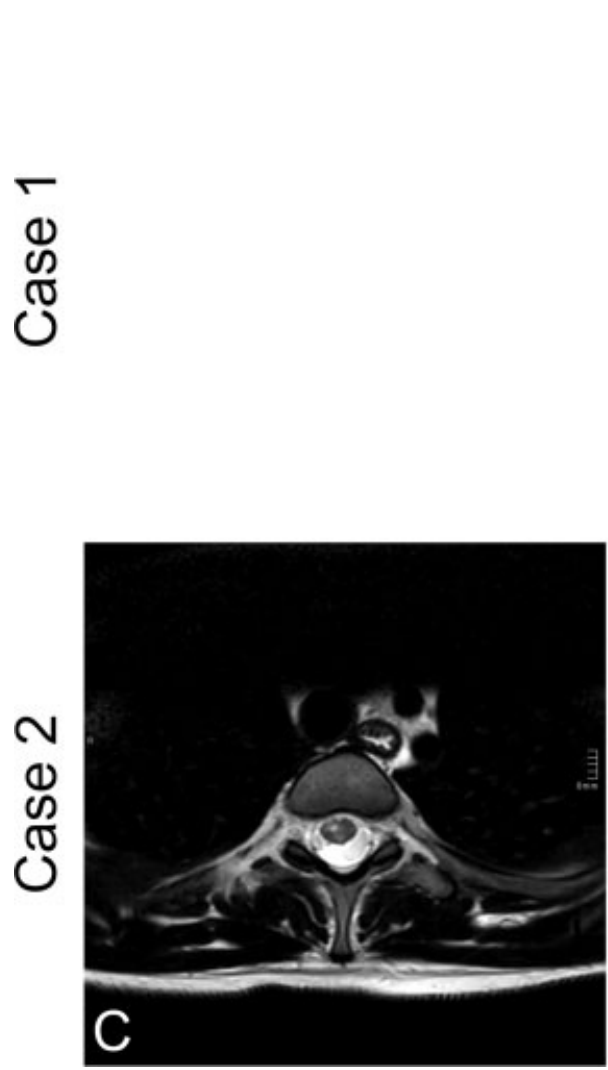

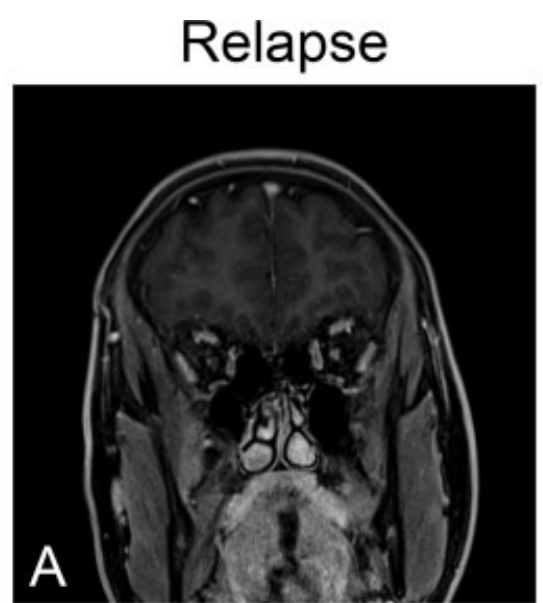

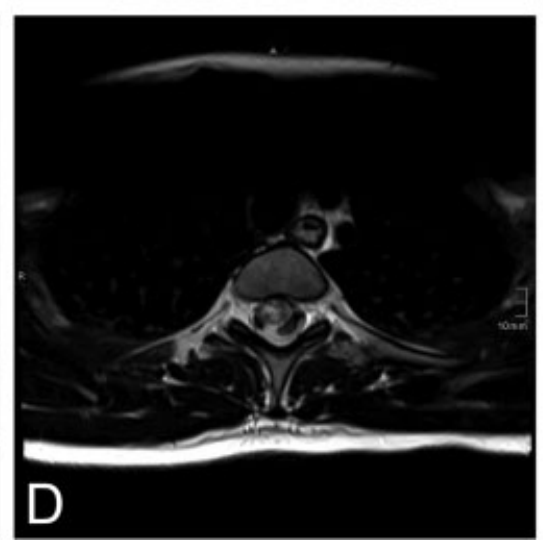

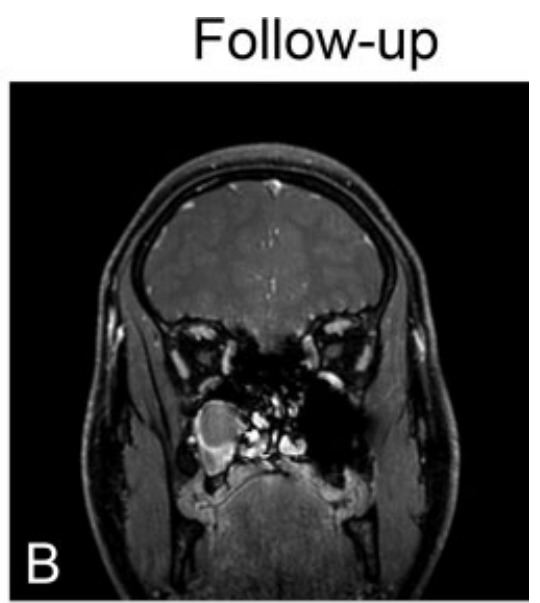

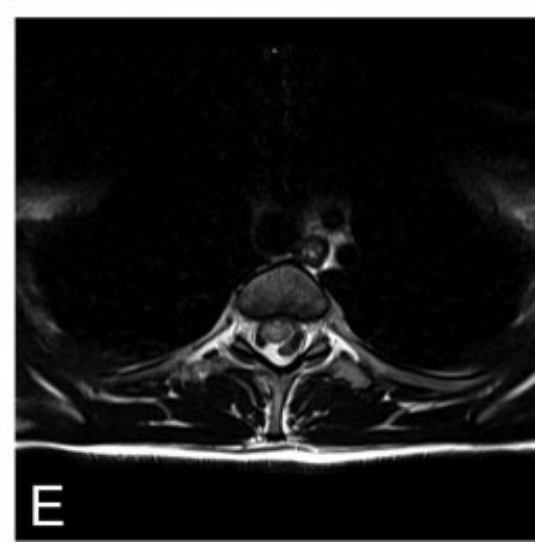

Fig. 2 MRI at onset, relapse, and follow-up. Coronal T1 images of case 1 (A, B) showing resolution of contrast enhancing lesion in the left optic nerve. Axial spinal T2 images of case 2 (C, D, E) showing increase of LETM at relapse and subsequent resolution until follow-up. LETM, longitudinally extensive transverse myelitis; MRI, magnetic resonance imaging.

Despite the absence of clinical signs of an acute attack of optic neuritis (no ocular pain, no acute/subacute onset), the patient reported a slowly progressive decrease of visual acuity during the second year of treatment. Twenty-four months after initiation of tocilizumab a decrease in visual acuity from 1.0 to 0.6 was noted in the left eye; however, atrophy measured by OCT had remained stable. Follow-up MRI revealed atrophy of both optic nerves (-Fig. 2) and residual subcortical lesions; however, no new intracranial or intraspinal lesions were found (-Fig. 1). At the latest clinical follow-up, the patient had remained relapse-free for 36 months after initiation of tocilizumab (EDSS 2.0). PLEX had led to a significant decrease of AQP4-ab titers (1:320, before initiation of tocilizumab) with subsequent gradual increase over the following months (1:5,120, 12 months after initiation of tocilizumab).

\section{Case 2}

A previously healthy 17-year-old girl presented with acute onset of paresthesia of both legs that increased with exercise. Three days later she described an additional severe pruritus on the back. Initial MRI showed LETM, but no intracranial abnormalities (-Fig. 1). CSF analysis revealed a mild pleocytosis with negative oligoclonal bands. In serum, AQP4-abs $(1: 10,240)$ were detected using live cell-based assays. Treatment with IVMP (30 mg/kg; 3 days) was initiated which led to resolution of pruritus and paresthesia. In the following days, the patient started suffering from anesthesia of the abdomen and the front of both thighs that resolved without treatment within 3 weeks. One month later the patient relapsed with paresthesia of chest and both legs as well as cramps of the right leg that increased on movement (EDSS 2.0). No visual deficits were noted. Treatment with IVMP, PLEX (8x), oral prednisolone tapering, and rituximab (375 $\mathrm{mg} / \mathrm{m}^{2}$ BSA, 4 weeks interval) was started which led to improvement of symptoms. One month later the patient relapsed with paresthesia and pruritus of the left chest and shoulder while under complete B cell depletion as measured by flow cytometric methods. Spinal MRI showed significant disease progression with multiple new intramedullary lesions (-Figs. 1 and 2). After additional PLEX (4x), therapy with tocilizumab ( $8 \mathrm{mg} / \mathrm{kg}$, monthly) was started which led to clinical stabilization. Follow-up MRI revealed residual signal changes, but no new lesions (-Figs. 1 and 2). At the latest clinical follow-up, the patient had remained relapsefree for 18 months after initiation of tocilizumab (EDSS 1.0). The AQP4-ab titers had not changed significantly.

\section{Discussion}

We report two female adolescents with AQP4-abs positive NMOSD who clinically stabilized after switching treatment 
from rituximab to monthly administrations of tocilizumab. Despite the efficacy of rituximab, up to $30 \%$ of patients suffer from clinical relapses in spite of complete B cell depletion. ${ }^{1-3}$ Pathophysiologically, CD20-depletion resistant plasmablasts have been proposed as origin of AQP4-abs in rituximab therapy resistant cases. Chihara et al identified $\mathrm{CD} 19^{\text {int }} \mathrm{CD} 27^{\text {high }} \mathrm{CD} 38^{\text {high }} \mathrm{CD} 18^{-}$plasmablasts in NMOSD that secrete AQP4-abs in an IL-6 dependent manner. ${ }^{5}$ In addition, they reported increased levels of IL- 6 in NMOSD relapses. Therefore, targeted therapy with the IL-6-blocking antibody tocilizumab has been suggested as an alternative to standard treatment. ${ }^{5}$

The escalation of therapy in case of rituximab therapy refractory NMOSD is a matter of debate. Our two patients were switched to tocilizumab 1 and 3 months, respectively, after initiation of rituximab treatment and confirmed B cell depletion. Some authors have described a time lag between B-cell depletion and clinical stabilization of NMOSD patients treated with rituximab. In such cases, a bridging therapy with, for example, low-dose corticosteroids has been proposed. ${ }^{6}$ Another option would be to switch to one of the widely used steroid-sparing drugs mycophenolate mofetil or azathioprine. A multicenter study comparing treatment efficacy of rituximab, azathioprine, and mycophenolate mofetil in NMOSD showed the highest reduction of relapse rate in patients treated with rituximab. ${ }^{7}$ In case of treatment failure switching to either azathioprine or mycophenolate mofetil led to a significant reduction in relapse rate. ${ }^{7}$ Mycophenolate mofetil and azathioprine are known to show a delay in clinical efficacy of several months. Due to the high clinical and paraclinical disease activity in both patients, the risk of further disabling relapses was considered high. Therefore, we decided to switch to tozilizumab.

Clinical data on tocilizumab treatment in NMOSD is sparse and has only been reported in adults in small case series and case reports. A current clinical trial (NCT02028884), including patients from 12 years of age aims at evaluating the efficacy and safety of satralizumab (SA237), another IL-6-blocking antibody, as add-on in the treatment of NMOSD. In terms of pediatric populations, only a single case of a 14-year-old male patient suffering from Sjögren's syndrome and NMOSD has been reported who became relapse-free after switching to tocilizumab. ${ }^{8}$

While previous reports in adults have shown mild recurrence in some patients, no relapses were seen in our two cases. This might be due to adaptation of administration frequency and dosing to recommendations published in a recent report ${ }^{9}$ ( 4 weekly infusions and $8 \mathrm{mg} / \mathrm{kg}$, respectively). However, one of our patients showed slowly progressive decrease in visual acuity during treatment without clinical signs of acute attack or further deterioration of OCT.
In terms of safety, no severe adverse effects or increased frequency of infections have been noted in our patients.

We conclude that early switching to tocilizumab might pose a viable treatment option in B cell-depletion resistant pediatric NMOSD. Larger studies are necessary to reliably evaluate efficacy and safety and define the patient groups who will profit most from this treatment.

\section{Patient Consent}

Written informed consent for patient information and MR images to be published was provided by the patients. The study was approved by the institutional review board (Ethikkomission der Medizinischen Universität Wien, No. EK1123_2015).

\section{Study Funding}

This study was supported in part by the grant "Jubiläumsfonds der Österreichischen Nationalbank, Project 16919" to R.H.

\section{Conflict of Interest}

None.

\section{References}

1 Jacob A, Weinshenker BG, Violich I, et al. Treatment of neuromyelitis optica with rituximab: retrospective analysis of 25 patients. Arch Neurol 2008;65(11):1443-1448

2 Pellkofer HL, Krumbholz M, Berthele A, et al. Long-term follow-up of patients with neuromyelitis optica after repeated therapy with rituximab. Neurology 2011;76(15):1310-1315

3 Kim S-H, Kim W, Li XF, Jung IJ, Kim HJ. Repeated treatment with rituximab based on the assessment of peripheral circulating memory B cells in patients with relapsing neuromyelitis optica over 2 years. Arch Neurol 2011;68(11):1412-1420

4 Araki M, Aranami T, Matsuoka T, Nakamura M, Miyake S, Yamamura T. Clinical improvement in a patient with neuromyelitis optica following therapy with the anti-IL-6 receptor monoclonal antibody tocilizumab. Mod Rheumatol 2013;23(04): 827-831

5 Chihara N, Aranami T, Sato W, et al. Interleukin 6 signaling promotes anti-aquaporin 4 autoantibody production from plasmablasts in neuromyelitis optica. Proc Natl Acad Sci U S A 2011; 108(09):3701-3706

6 Hinson SR, Lennon VA, Pittock SJ. Autoimmune AQP4 channelopathies and neuromyelitis optica spectrum disorders. Handb Clin Neurol 2016;133:377-403

7 Mealy MA, Wingerchuk DM, Palace J, Greenberg BM, Levy M. Comparison of relapse and treatment failure rates among patients with neuromyelitis optica: multicenter study of treatment efficacy. JAMA Neurol 2014;71(03):324-330

8 Marino A, Narula S, Lerman MA. First pediatric patient with neuromyelitis optica and Sjögren syndrome successfully treated with tocilizumab. Pediatr Neurol 2017;73:e5-e6

9 Ayzenberg I, Kleiter I, Schröder A, et al. Interleukin 6 receptor blockade in patients with neuromyelitis optica nonresponsive to anti-CD20 therapy. JAMA Neurol 2013;70(03):394-397 